\title{
Directional Constraint Evaluation in Optimality 'Theory*
}

\author{
Jason Eisner \\ Department of Computer Science / University of Rochester \\ Rochester, NY 14607-0226 (U.S.A.) / jason@cs.rochester. edu
}

Weighted finite-state constraints that can count unboundedly many violations mako Optimality Theory more powerful than finite-state transduction (Frank and Satta, 1998). This result is empirically and compulationally awkward. We propose replacing these mibounded constmants, as well as non-finite-states Generalized Aligmmont constraints, with a new class of finito-state dircctional constrainls. Wo give linguistic applications, results on generative power, and algorithms to compile granmmars into transducers.

\section{Introduction}

Optimality Thoory is a grammar fianework that directly expresses comstrantint on phonological forms. Rowghly, the grammar prefers forms that violate cach constraind as little as possible.

Most constraints used in pratice describe disfavored local configurations in the phonological form (Fisner, 1997a). It is therefore possible for a given form to oflend a single constiant at several localions in the form. (lor oxample, a constraint against syllable codas will be offonded by every syllable lhat has a cocha.)

When comparing forms, then, how do we aggregate a form's multiple local oflensess into an overall violation level?

$\Lambda$ constraint conld answer this question in at leasti three ways, the third boing our proposal:

- Unbounded evaluation (Prince and Smolensky, 1993). A form's violation level is given by the number of offenses. Forms with fewer oflenses are prefered.

- Bounded evaluation (Frank and Satia, 1998; Karttumen, 1998). A forn's violat tion level is min( $k$, number of offonses $)$ for some k. This is like unbounded evaluation except that the constraint does not; distinguish among forms with $\geq k$ oflenses. ${ }^{1}$

- Directional evaluation. A form's violation level considers the location of offonses, not their total number. Under left-

* I am grateful to the 3 anonymous referes lor fectlback.

${ }^{1}$ Note that $k-1$ gives "binary" constraints that can be described simply as langnages. Any $k$-bounded constraint can casily be simuladed by $k$ : binary constraints. to-right evaluation, the constraint prefers forms whose offenses are as late as possible. 'Io compare two forms, it aligns then (accorcling to their common underlying representiation), and scans them in parallel from left to right, stopping at the first location where one form has an offense and the other does not ("sudden death"): it profer's the latice. Right-to-left cvaluation is similar.

$\$ 2$ of this paper gives linguistic and computational notivation for the proposal. \$3 formalizes the idea and slows that composing a transducer. with a dincetional constraint yields a transducer. Thus directional comstraints, like bounded ones, keep or within the class of regular relations. (but, we also show them to be more oxpressive.)

\section{Motivation}

\subsection{Intuitions}

Recall that O'l's constiraint ranking mechanism is an answer to tho question: How can a grannmatr evaluale a form by aggregating its violattions of several constraints? Above we asked the same question at a finer scale: How can a constratnt, evaluate a form by aggregating its offonses at several locations? Figure 1 illustrates that our answer is just constraint ranking ledux.

Directional evaluation strictily ranks the importance of the locations within a form, e.g., from left to right. "This excmplities OT"s "do only when necessary" strategy: the constraint prefors to postpone offenses until they becomo strictly necessary toward the right of the form, even at the cost of having more of them.

One might, think from Figure 1. lhat each directional constraint conld be decomposed into several binary or other bounded constraints, yiclling a granmar using only bounded constraints. However, no single such grammar is general enough to handle all inputs: the number of constraints needed for the decomposition corresponds to the Iength (i.c., the number of locations) of the nuderlying representation. 


\begin{tabular}{l|}
\hline ban.to.di.bo \\
\hline ban.ton.di.bo \\
\hline ban.to.dim.bon \\
\hline ban.ton.dim.bon
\end{tabular}

\begin{tabular}{l||l|l|} 
(a) & $C_{1}$ & NoCODA \\
\hline-1 & $* !$ & $*$ \\
\hline wat & & $* *$ \\
\hline & & $* * * !$ \\
\hline & & $* * * ! *$ \\
\hline
\end{tabular}

\begin{tabular}{l||l|l|l|l|l|}
$(\mathrm{b})$ & $C_{1}$ & $\sigma_{1}$ & $\sigma_{2}$ & $\sigma_{3}$ & $\sigma_{1}$ \\
\hline \hline & $* !$ & $*$ & & & \\
\hline & & $*$ & $* !$ & & \\
\hline as & & $*$ & & $*$ & $*$ \\
\hline & & $*$ & $* !$ & $*$ & $*$ \\
\hline
\end{tabular}

\begin{tabular}{l||l|l|l|l|l|} 
(c) & $C_{1}$ & $\sigma_{4}$ & $\sigma_{3}$ & $\sigma_{2}$ & $\sigma_{1}$ \\
\hline \hline & $* !$ & & & & \\
\hline I 35 & & & & $*$ & $*$ \\
\hline & & $* !$ & $*$ & & $*$ \\
\hline & & $* !$ & $*$ & $*$ & $*$ \\
\hline
\end{tabular}

Figure 1: Directional evaluation as subconstraint ranking. $A 11$ candidates have 4 syllables; we simplify here by regarding these as the locations. $C_{1}$ is some high-ranked constraint that eliminates ban.bo.di.bo; NoCoNA is offended by syllable codas. (a) Traditional unbounded evaluation of NOCODA. (b) Left-to-right evaluation of NoCoDA, shown as if it were split into 4 constraints evaluating the syllables separately. (c) Righti-to-left.

\subsection{Iterative and floating phenomena}

The main empirical motivation for dircetionally evaluated constraints is the existence of "iterative" phenomena such as motrical footing. (Derivational theories described these with procedures that scannod a form from one end to the other and modified it; sce (Johnson, 1972).)

for most other phenomena, directional constraints are indistinguishable from traditional unbounded constraints. Usually, the candidates with the lewest offenses are still the ones that survive. (Since their competitors offend at exactly the same locations, and more.) This is precisely because most phonology is local: satiisfying a constraint at one location does not usually block satisfying it at another.

Distinguishing cases, like the artificial Fig. 1---where the constraint can only trade offenses at one location for offenses at another arise only under special conditions involving nomlocal phenomena. Just as directional evaluation would predict, such a forced tradeoff is always resolved ( to our knowledge) by placing oflenses as late, or as carly, as higher constraints allow:

- Prosodic groupings force each segment or syllable to choose which constituent (if any) to associate with. So-called left-toright directional syllabification (Mester and Padgett, 1994) will syllabify /CVCCCV/ greedily as CVC.CV.CV rather than CV.CVC.CV, postponing epenthetic material until as late as possible. Similarly, left-to-right binary footing (Hayes, $1995)$ prefers $(\sigma \sigma)(\sigma \sigma) \sigma$ over $\sigma(\sigma \sigma)(\sigma \sigma)$ or $(\sigma \sigma) \sigma(\sigma \sigma)$, postponing unfooted syllables.

- Floating lexical material must, surface somewhere in the form. Floating features (e.g., tone) tend to dock at the leftmosti or rightmost available site, postponing the appearance of these marked feat,ures. Infixed morphemes tend to be infixed as little as possible (McCarthy and Prince, 1995), postponing the appearance of an affix edge or other affix material within the stem. ${ }^{2}$

- Floating non-lcxical matcrial must also appear somewhere. If a high-ranked constraint, Culminativity, requires that a primary stress mark appcar on cach word, then a directional constraint against primary stress will not only prevent additional marks but also push the single mark to the first or last available syllable the traditional "End Rnle" (Prince, 1983).

- Harmony must decide how lar to spread features, and $O C P^{\prime}$ effects such as Grassman's Law must decide which copies of a feature to eliminatice. Again, directional evaluation seems to capture the facts.

\subsection{Why not Generalized Alignment?}

In OT, following a remark by Robert Kirchner, it has been traditional to analyze such phenomena using highly non-local Generalized Alignment (GA) constraints (McCartly and Prince, 1993). For cxample, left-l,o-right fooling is favored by $\Lambda_{I} I G N-L E F^{\prime} J_{W}$ (loot, Stem), which requires every foot to be lefi-aligned with a morphological stem. Not only does each misaligned foot offend the constraint, but the seriousness of its offense is given by the

\footnotetext{
2 " $\Lambda$ vailable site" and "possible" amount of infixation are defined here by higher-ranked constraints. These might restrict the allowed tone-bearing units and the allowed CV shape after infixation, but do not fully determine where the floating material will surface.

A referce asks why codas do not also float (to postpone NoCoda offenses). Answer: Flotation requires unusual, non-local mechanisms. Gen or a constraint must cnsure that an anchored tone sequence resembles the underlying floating tone sequence, which may be represented on an anxiliary input tape or (if bounded) as an input prefix. But ordinary faithfulness constraints check only whether underlying material surfaces locally; they would penalize coda flotation as a local deletion plus a local insertion.
} 
mumber of syllables by which it is misaligned. 'T]ese 1 mmbers are simmed over all oflending? feet to obtain the violation level. Hor exanplo, $[\sigma(\sigma \sigma)(\sigma \sigma) \sigma(\sigma o)]_{\text {sicm }}$ has $1+3-+6-10$ violations, and $[\sigma \sigma \sigma \sigma(\sigma \sigma)(\sigma \sigma)]_{\text {stem }}$ is equally bad at $4+6=10$ violations. Shifting fect leftwand or eliminaling then reduess the violation level.

(Stemberger, 1996) argued that GA comstanints wore too powerful. (Bllison, J995) showed that; no single finite-state nulyomeled constraint conld define the sime violation levols as a (G $\Lambda$ constrant. (19isner, 1997a) slowed more strongly that since $G A$ an be made to center a floating tome on a phrase," no hararchy of finite-state monounded constraints could even define the sane optimal comdidetes as a GA constrant. 'I'lum GA cammot be simmlated in Filison's (1994) finite-state famework (83.2).

for this reason, as well ats the awkwarlness aud non-locality of evaluating $G \wedge$ oflenses, wo propose to replace ( $\mathrm{x} A$ with dinectional con-

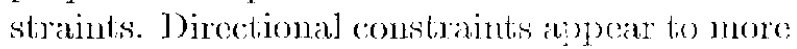
divectly saptiure the obsereved phomomenta.

We do note that another, triekier possibility is

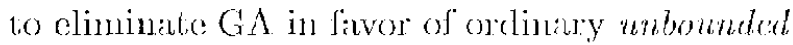
constraints tiant are indifferent to the location of oflenses. (Fjlison, 1994) noted that GA constraints that coaluated the placemont of only one eloment (e.g., primary stress) conld be leplaced by simpler NoINTEliventNe constraints. (Tisner, 1997b) gives a GA-free treatument of the metrical stiress typology of (Hayes, 1995).

\subsection{Generative power}

It has recently been proposed that for compontational reasons, O'T shomld eliminate notionly GA but all unbounded constraints (Fraus and Satta, 1998; Karttunen, 1998). As with GA, we offer the less extreme approach of replacing thom with directional constraints instoad.

Recall that a phonological granmar, as 11sually conceived, is a description of permissible (UR, SlR) pairs." It has long been bolicved tiat naturally occurring phomological ganminats are regular relations (Johnson, 1972; Kaplan and Kay, 1994). 'This moans that they can be implenented as finitc-state transducers (FS'Is) that accept exactly the grammatical pairs. FSTs are immensely usefiul in perfornu-

\footnotetext{
${ }^{3}$ This is indeed too powerful: centering is mattested. ${ }^{n} \mathrm{UR}=$ underlying representation, $\mathrm{SR}=$ surlace repn .
}

ing many rolevant lasks rapidly: goneration (ob) laining all possible S]R for a URR), complehension (conversely), charaterizing the set of form on which two grammars (perhaps from different descriptive frameworks) would differ, ete. Moreover, FS't's an bo applied in parallel to regular sets of forms. lor example, one can obtain a woighted set of possible Slis (a phoneme lattice) from a spexch recognizer, pass it through the inverse transducer, intersect the resulting weighter sel of URs with the lexicon, and then extract the hest, surviving URs.

(Fllison, 1994; Jisner, 1997a) frame OT within this tadition, by modeling Gen and the constiraintis as weighted finite-state nuachiness (sec 33.2$)$. But althongh those papers showed how to generabe the sel of STs lor a single given UlR, they dirl not compilo tho oT' grammar into anl $\mathrm{J}^{\prime} \mathrm{S}^{\prime} \mathrm{J}^{\prime}, \mathrm{y}$ obtain the other benefits thereof.

In fact, (Jank and Salta, 1998) showed that, such compilation is impossible ju the general case of mbomeded constraints. 'lo soo why, consider the grammal: MAx, JHes, II Al-

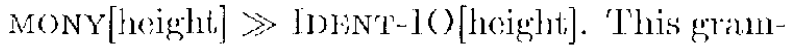
mat: insisto on hoight hasnony anong sustace vowels, but dislikes changes from the UR. 'Ihe resulti is the mattested phemomonon of "majorily assimilation" (Bakovié, 1999); Lombardi, 1999): a UlR with more high vowols than low will surface with all vowels high, and vice-versal. So O't nuay compore unboundal counts in a way that an lis' cammot, and plomology doos not.

'Jhis suggests that; (O'l' with unbounded constaraints is too powerfirl. Jence (tibank and Saltia, 1998; Kartituncu, 1998) propose using only boumded constrantis. 'They show this roduces OT's power to finite-state mansduction.

The worry is that bounded constraints may not. be expressive enough. A 2-bonnded version of NoComa would not distinguish among the final three forms in Figure 1: it is agnostis: when the input forces multiple codas in all candidates.

'To be sure, a k-bounded approximation may work well for large $k .5$ l3ut its automaton (83.2) will typically have $k$ times as many states as the mbounded original, since it unolls loops: the

\footnotetext{
${ }^{5}$ Using the approximate grammar for gencration, an oubput is gunanteed correct unless it aclieves $k$ violations for some $k$-bounded constraint. One can then raise $k$, recomple the grammar, and try again. But $k$ may grow quite large for long inputs like phonological phrases.
} 
state must keep track of the offense count. Intersecting many such large constraints can produce very large FSTs - while still failing to capture simple generalizations, c.g., that all codas arc dispreferred.

In $\S 3$, we will show that directional constraints are more powerful than bounded constraints, as they can express such generalizations-yet they keep us within the world of regular relations and FS'Ts.

\subsection{Related Work}

Walther (1999), working with intersective constraints, defines a similar notion of Bounded Local Optimization (BLO). Trommer (1998; 1999) applies a variant of Walther's idea to OT. The motivation in both cases is linguistic.

We sketch how our idea differs via 3 examples:

\begin{tabular}{rl|ll|l}
$U R$ & uuuuu & uu & uuu & uuuuur \\
candidate $X$ & vvvbb & vv & vbb & vvvbb \\
candidate $Y$ & vvbaa & vvvvbaa & vzbaa \\
\hline
\end{tabular}

Consider $* b$, a left-to-right constraint that is offended by cach instance of $b$. On our proposal, candidate $\mathrm{X}$ wins in each column, because $\mathrm{Y}$ always offends $* b$ first, at position 3 in the UR.

But under BLO, this offense is not fatal. Y can survive $* b$ by inserting epenthetic material (column 2: $\mathrm{Y}$ wins by postponing $b$ relative to the SR), or by changing $v$ to $z$ (columm 3: Y ties $\mathrm{X}$, since $v v \neq v z$ and BLO merely requires the cheapest choice given the surface output so far). In the same way, NoCoDA under BLO would trigger many changes unrelated to codas. Our definition avoids these apparent inconveniences.

Walther and Trommer do not consider the expressive power of BLO (cf. \$3.3) or whether grammars can be compiled into UR-to-SR FSTs (our main result; see discussion in $§ 3.4$ ).

\section{Formal Results}

\subsection{Definition of OT}

An OT grammar is a pair (Gen, $\vec{C}$ ) where

- the candidate generator Gen is a relation that maps each input to a nonempty set of candidate outputs;

- the hierarchy $\vec{C}=\left(C_{1}, C_{2}, \ldots\right)$ is a finite tuple of constraint functions that evaluate outputs.

We write $\vec{C}(\delta)$ for the tuple $\left(C_{1}(\delta), C_{2}(\delta), \ldots\right)$.
Given a UR, $\sigma$, as input, the grammar admits as its SRs all the outputs $\delta$ such that $\vec{C}(\delta)$ is lexicographically minimal in $\{\vec{C}(\delta): \delta \in \operatorname{Gen}(\sigma)\}$.

The values taken by $C_{i}$ are called its violation levels. Conventionally these are natural numbers, but any ordered set will do.

Our directional constraints require the following innovations. Each input $\sigma$ is a string as usual, but the outputs are not strings. Rather, each candidate $\delta \in \operatorname{Gen}(\sigma)$ is a tuple of $|\sigma|+1$ strings. We write $\bar{\delta}$ for the concatenation of these strings (the "real" SR). So $\delta$ specifies an alignment of $\bar{\delta}$ with $\sigma$. The directional constraint $C_{i}$ maps the tuple $\delta$ to a tuple of natural numbers ("offense levels") also of length $|\sigma|+1$. Its violation levols $\left\{C_{i}(\delta): \delta \in \operatorname{Gen}(\sigma)\right\}$ are compared lexicographically.

\section{$3.2 \quad$ Finite-state assumptions}

Wo now confine our attention to finite-state OT grammars, following (Ellison, 1994; Tesar, 1995; Eisner, 1997a; Frank and Satta, 1998; Karttunen, 1998). Gen $\subseteq \Sigma^{*} \times \Delta^{*}$ is a regular relation, ${ }^{6}$ and may be implemented as an unweighted FST. Each constraint is implemented ${ }^{7}$ as a possibly nondeterministic, weighted finitestate automaton (WFSA) that accopts $\Delta^{*}$ and whose ares are weighted with natural numbers.

An FST, $T$, is a finitc-state automaton in which each arc is labeled with a string pair $\alpha: \gamma$. Without loss of generality, we require $|\alpha| \leq 1$. This lets us define an aligned transduction that maps strings to tuples: If $\sigma=a_{1} \cdots a_{n}$, we define $T(\sigma)$ as the set of $(n+1)$-tuples $\delta=\left(\delta_{0}, \delta_{1}, \ldots \delta_{n}\right)$ such that $T$ has a path transducing $\sigma: \bar{\delta}$ along which $\delta_{0} \cdots \delta_{i-1}$ is the complete output before $a_{i}$ is read from the input.

We now describe how to evaluate $C(\delta)$ where $C$ is a WFSA. Consider the path in $C$ that accepts $\bar{\delta}_{.}^{8}$ In (un)bounded evaluation, $C(\delta)$ is the total weight of this path. In left-to-right evaluation, $C(\delta)$ is the $n+1$ tuple giving the respective total weights of the subpaths that consume $\delta_{0}, \ldots \delta_{n}$. In right-to-left evaluation, $C(\delta)$ is the reverse of the previous tuple. ${ }^{9}$

\footnotetext{
${ }^{6}$ Ellison required only that $\operatorname{Gen}(\sigma)$ be regular $(\forall \sigma)$.

${ }^{7}$ Space prevents giving the equivalent characterization as a locally weighted language (Walther, 1999).

${ }^{8}$ If there are multiple accepting paths (nondeterminism), take the one that gives the least value of $C(\delta)$.

${ }^{9}$ This is equivalent to $C^{R}\left(\delta_{n}^{R}, \ldots, \delta_{0}^{R}\right)$ where ${ }^{R}$ denotes reversal of the automaton or string as appropriate.
} 


\subsection{Expressive power}

Thanks to Gen, finite-statio OT can trivially implement any regular inputi-output relation with no constraints at all! And $\$ 3.4$ bolow shows that whether: we allow directional or bounded constraints does not affect this generative power.

But in another sense, directional constraints are strictly more expressive than bounded ones. If Gen is fixed, then any hierarchy of bounded constraints can be simulated by some hierarchy of directional constraints ${ }^{10}$ but not vice-versa.

Indeed, we show even more strongly that directional constraints cannot always be simnlated even by unbounded constraints. ${ }^{11}$ Define $* b$ as in $\$ 2.5$. This ranks the set $(a \mid b)^{n}$ in lexicographic order, so it makes $2^{n}$ distinctions. Let Gen be the regular relation

$(a: a \mid b: b)^{*}\left(c: a(a: a \mid b: b)^{*} \mid c: b(a: a|b: b| a: b \mid b: a)^{*}\right)$

We claim that the grammar (Gen, $* b)$ is not equivalent to $\left(\mathrm{Gen}, C_{1}, \ldots, C_{s}\right)$ for any bounded or unbounded constraints $C_{1}, \ldots C_{s}$. There is some $k$ such that for all $\delta \in \Delta^{n}$, wach $C_{i}(\delta)<$ $k n .^{12}$ So candidates $\delta$ of length $n$ have at most $(k * n)^{s}$ different violation profiles $\vec{C}(\delta)$. Choose $n$ such that $2^{n}>(k n)^{s}$. Then the set of $2^{n}$ strings $(a \mid b)^{n}$ must contain two distinct strings, $\delta=$ $x_{1} \cdots x_{n}$ and $\delta^{\prime}=y_{1} \cdots y_{n}$, with $\vec{C}(\delta)=\vec{C}\left(\delta^{\prime}\right)$. Let $i$ be minimal such that $x_{i} \neq y_{i}$, and without loss of generality assume $x_{i}=a, y_{i}=b$. Put $\sigma=x_{1} \cdots x_{i-1} c x_{i+1} \cdots x_{n}$. Now $\delta, \delta^{\prime} \in \operatorname{Gen}(\sigma)$ and $\delta$ is lexicographically minimal in $\operatorname{Gen}(\sigma)$. So the grammar (Gen, $* b$ ) majs $\sigma$ to $\delta$ only, whereas (Gen, $\vec{C}$ ) cannot distinguish between $\delta$ and $\delta^{\prime}$, so it maps $\sigma$ to neither or both.

\subsection{Grammar compilation: $\mathrm{OT}=$ FST}

It is trivial to translate an arbitrary FST grammar into OT: let Gen be the FST, and $\vec{C}=()$. The rest of this section shows, conversely, how to compile a finite-state OT grammar (Gen, $\vec{C}$ ) into an FST, provided that the grammar uses only bounded and/or directional constraints.

\footnotetext{
${ }^{10}$ How? Jy using states to count, a bounded constraint's WISA can be transformed so that all the weight of each path falls on its final arc. This defines the same optinal candidates, even when interpreted directionally.

${ }^{11}$ Nor vice-versa, since only unbounded constraints can implement non-regular relations $(\$ 2.4, \$ 3.4)$.

${ }^{12}$ Apply $\$ 3.4 .4$ to eliminate any $\epsilon$ 's from the constraint WDFAs (regarded as outputless transducers), then take fo to exceed all arc: weights in the result.
}

\subsubsection{The outer loop of compilation}

Jet $T_{0}=$ Gen. For $i>0$, we will construct an FST $T_{i}$ that inplements the partial grammar (Gen, $C_{1}, C_{2}, \ldots C_{i}$ ). We construct $T_{i}$ from $T_{i-1}$ and $C_{i}$ only: $T_{i}(x)$ contains the forms $y \in T_{i-1}(x)$ for which $C_{i}(y)$ is minimal.

If $C_{i}$ is $k$-bounded, we use the construction of (Frank and Satta, 1998; Karttuncn, 1998).

If $C_{i}$ is a left-to-right constraint, we compose $T_{i-1}$ with the WFSA that represents $C_{i}$, obtaining a weighted finite-state transducer (WFST), $\hat{T}_{i}$. This transducer may be regarded as assigning a $C_{i}$-violation level (an $(|\sigma|+1)$-tuple) to each $\sigma: \delta$ it accepts. We must now prune away the suboptimal candidates: using the DBP algorithm below, we construct a new unweighted FST $T_{i}$ that transduces $\sigma: \delta$ iff the weighted $\hat{T}_{i}$ can transduce $\sigma: \delta$ as chcaply as any $\sigma: \delta^{\prime}$.

If $C_{i}$ is right-to-left, we do just the same, except DBP is used to construct $T_{i}^{R}$ from $\hat{T}_{i}^{h}$.

\subsubsection{Directional Best Paths: The idea}

All that remains is to give the construction of $T_{i}$ from $\hat{T}_{i}$, which we call Directional Best Paths (DBP). Recall standard best-paths or shortest-paths algorithms that pare a WFSA down to its patihs of minimum total weight (Dijkstra, 1959; Bllison, 1994). Our greedier version does not sum along paths but always inmediately takes the lightest "available" arc.

Crucially, available ares are defined relative to the input string, because we must retain one or more optimal output candidates for each input. So availability requires "lookahead": we must take a heavier arc $(b: x$ bolow) just when the rest of the input (c.g., abd) cannot otherwise be accepted on any path.

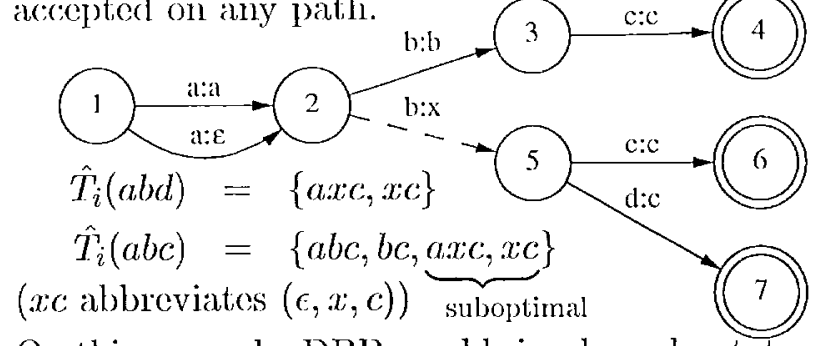

On this example, DBP would simply make state 6 non-final (forcing abe to take the light arc unavailable to $a b d)$, but often it must add states!

This rolativization is wlat lets us compile a hierarchy of directional constraints, once and for all, into an single FST that can find the optimal output for any of the infinitely many possible inputs. We saw in $\$ 2.4$ why this is so desirable. By 
contrast, Ellison's (1994) best-paths construction for unbounded constraints, and previously proposed constructions for directional-style constraints (sce $\$ 2.5$ ) only find the optimal output for a single input, or at best a finite lexicon.

\subsubsection{Dir. Best Paths: A special case}

$\$ 3.2$ restricted our FSTs such that for every arc label $\alpha: \gamma,|\alpha| \leq 1$. In this section we construct $T_{i}$ from $\hat{T}_{i}$ under the stronger assumption that $|\alpha|=1$, i.e., $\hat{T}_{i}$ is $\epsilon$-free on the input side.

If $Q$ is the stateset of $\hat{T}_{i}$, then let the stateset of $T_{i}$ be $\{[q ; R ; S]: R \subseteq S \subseteq Q, q \in S-R\}$. This has size $|Q| \cdot 3^{|Q|-1}$. However, most of these states are typically umreachable from the start state. Lazy "on-the-fly" construction techniques (Mohri, 1997) can be used to avoid allocating states or arcs until they are discovered during exploration from the start state.

For $\sigma \in \Sigma^{*}, q \in Q$, define $V(\sigma, q)$ as the minimum cost (a $|\sigma|$-tuple of weights) of any $\sigma$-reading path from $\hat{T}_{i}$ 's start state $q_{0}$ to $q$.

The start state of $T_{i}$ is $\left[q_{0} ;() ;\left\{q_{0}\right\}\right]$. The intent is that $T_{i}$ have a path from its start state to $[q ; R ; S]$ that transduces $\sigma: \delta^{13}$ iff

- $\hat{T}_{i}$ has a $q_{0}$ to $q, \sigma: \delta$ path of cost $V(\sigma, q)$;

- $R=\left\{q^{\prime} \in Q: V\left(\sigma, q^{\prime}\right)<V(\sigma, q)\right\}$; and

- $S=\left\{q^{\prime} \in Q: V\left(\sigma, q^{\prime}\right) \leq V(\sigma, q)\right\}$.

So as $T_{i}$ reads $\sigma$, it "follows" $\hat{T}_{i}$ 's cheapest $\sigma$ reading paths to $q$, while calculating $R$, to which yet cheaper (but perhaps dead-end) paths exist.

Let $[q ; R ; S]$ be a final state (in $T_{i}$ ) iff $q$ is final and no $q^{\prime} \in R$ is final (in $\hat{T}_{i}$ ). So an accepting path in $\hat{T}_{i}$ survives into $T_{i}$ iff there is no lowercost accepting path in $\hat{T}_{i}$ for the same input.

The arcs from $[q ; R ; S]$ correspond to arcs from $q$. For each arc from $q$ to $q^{l}$ labeled $a: \gamma$ and with woight, $W$, add an unweighted $a: \gamma$ arc from $[q ; R ; S]$ to $\left[q^{\prime} ; R^{\prime} ; S^{\prime}\right]$, provided that the latter state exists (i.c., unless $q^{\prime} \in R^{\prime}$, indicating that there is a cheaper path to $q^{\prime}$ ). Here $R^{\prime}$ is the set of states that are either reachable from $R$ by a (single) $a$-reading arc, or reachable from $S$ by an $a$-reading arc of wcight $<W$. $S^{\prime}$ is the union of $R^{\prime}$ and all states reachable from $S$ by an $a$-reading arc of weight $W$.

\subsubsection{Dir. Best Paths: The general case}

To apply the above construction, we must first transform $\hat{T}_{i}$ so it is $\epsilon$-frce on the input side. Of

\footnotetext{
${ }^{13} \delta$ is a tuple of $|\sigma|+1$ strings, but $\delta_{0}=\epsilon$ by $\epsilon$-freeness.
}

course input $\epsilon$ 's are crucial if Gen is to be allowed to insert unbounded amounts of surface material (to be pruned back by the constraints). ${ }^{\top 4}$ To eliminate $\epsilon$ 's while preserving these semantics, we are forced to introduce FST arc labels of the form $a: \Gamma$ where $\Gamma$ is actually a regular set of strings, represented as an FSA or regular expression. Following c-elimination, we can apply the construction of $\$ 3.4 .3$ to get $T_{i}$, and finally convert $T_{i}$ back to a normal tiransducer by expanding each $a: \Gamma$ into a subgraph.

When we eliminate an arc labeled $\epsilon: \gamma$, wo must push $\gamma$ and the arc's weight back onto a previous non- $\epsilon$ arc (but no further; contrast (Mohri, 1997)). The resulting machine will implement the same aligned transduction as $\hat{T}_{i}$ but more transparently: in the notation of $\$ 3.2$, the arc reading $a_{i}$ will transduce it directly to $\delta_{i} .{ }^{15}$

Concretely, suppose $\hat{T}_{i}$ can get from state $q$ to $q^{\prime \prime}$ via a path of total weight $W$ that begins with $a: \gamma_{1}$ on its first arc followed by $\epsilon: \gamma_{2}$, $\epsilon: \gamma_{3}, \ldots$ on its remaining arcs. We would like to substitute an arc from $q$ to $q^{\prime \prime}$ with label $a: \gamma_{1} \gamma_{2} \gamma_{3} \ldots$ and weight $W$. But there may be infinitely many such $q-q^{\prime \prime}$ paths, of varying weight, so we actually write $a: \Gamma$, where $\Gamma$ describes just those $q-q^{\prime \prime}$ paths with minimum $W$.

The exact procedure is as follows. Let $G$ be the possibly disconnected sulgraph of $\hat{T}_{i}$ formed by $\epsilon$-reading arcs. Run an all-pairs shortestpaths algorithm ${ }^{16}$ on $G$. This finds, for each state pair $\left(q^{\prime}, q^{\prime \prime}\right)$ connected by an $\epsilon$-reading path, the subgraph $G_{q^{\prime}, q^{\prime \prime}}$ of $G$ formed by the minimum-weight $\epsilon$-reading paths from $q^{\prime}$ to $q^{\prime \prime}$, as well as the common weight $W_{q^{\prime}, q^{\prime \prime}}$ of these paths. So for each arc in $\hat{T}_{i}$ from $q$ to $q^{\prime}$, with weight $W$ and label $a: \gamma$, we now add an arc to $T_{i}$ from $q$ to $q^{\prime \prime}$ with weight $W+W_{q^{\prime}, q^{\prime \prime}}$ and label $a: \gamma G_{q^{\prime}, q^{\prime \prime}}(\epsilon)$. $(G(\epsilon)$ denotes the regular language to which $G$ transduces $\epsilon$.) Having done this, wo can delete all $\epsilon$-roading arcs.

The modified $\epsilon$-free $\hat{T}_{i}$ is equivalent to

\footnotetext{
${ }^{14}$ As is conventional. Besides epenthetic matcrial, Gen often introduces copious prosodic structure.

${ }^{15}$ That arc is labeled $a_{i}: \Gamma$ where $\delta_{i} \in \Gamma$. But what is $a_{0}$ ? A special symbol $E \in \Sigma$ that we introduce so that; $\delta_{0}$ can be pushed back onto it: Before $\epsilon$-elimination, we modify $\hat{T}_{i}$ by giving it a new start state, comnected to the old start state with an are $E: \epsilon$. After $\epsilon$-climination, we apply DBP and replace $E$ with $\epsilon$ in the result $T_{i}$.

${ }^{16}$ (Cormen et al., 1990) cites several, including fast algorithms for when edge weights are small integers.
} 
the original except for eliminating some of the suboptimal subpaths. Here is a graph fiagment before and after c-elimination:

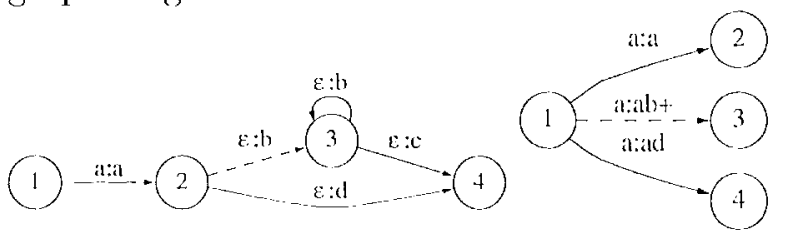

Note: Right-to-left evaluation applies I)131 to $\hat{I}_{i}^{R}$, so consistency with our previous definitions means it must push $c$ 's forwarel, not backward.

\section{Conclusions}

This paper has proposed a new notion in ('T: "directional evaluation," where underlying locations are strictly ranked by their importance.

'Traditional finite-state O'T. constraints have enough power to compare arbitarily high counts; Generalized Alignment is even worse. Directional constraints seom to captine the puros of these constraints: they appropriately militate against; every iustiance of a disfavored configuration in a andelidate form, no matter how many, and they naturally captine iterative and edgenost effocts. Yet they do not have the oxcess power: wo have shown that; an grammar of directional and/or bonnded constraints can be compiled into a finite-state transducer. That is both empirically and computationally desirable.

The most obvious future work comes from lingristics. Can directional constraints do all the work of unbounded and GA constraints? How do they change the style of amalysis? (I.g., directional versions of markedness constraints piu down the locations of marked objects, leaving lower-ranked constraints no say.) Finally, direc;tional constraints can be varionsly formulated (is * CIUSTER offended at the stiart or end of: cach cluster? or of its enclosing syllable?). So what conventions or restrictions should apply?

\section{References}

Eric Bakovic. 1999. Assimilation to the mmarked. Ms., Rutgers Optimality Archive ROA-340.

T. H. Cormen, C. E. Leiserson, and R. L. Rivest. 1990. Introduction to Algorithms. MIT Press.

Edsger W. Jijkstra. 1959. A note on two problems in connexion with graphs. Numerische Mathematik, 1:269-271.

Jason Eisner. 1997a. Efficient generation in prinitive Optimality Theory. In Proc. of the 35 th $A n$ nual $A C L$ and 8th FACL, Madrid, July, 313320.

Jason Eisner. 1997b. Foot Tolum decomposed: Us- ing primitive constraints in O'I'. In Bonjannin Brotening, editor, Proce of SCIL VIII, MIT Working Papers in Iinguistics 31, Cambridge, MA.

'T'. Mark Ellison. 1994. J'honological derivation in optimality theory. In Proce of COLING.

I'. Mark Ellison. 1995. (O'J, finite-state representations and procedurality. In Proc. of the Conference on Itormal Grammar, Barcelona.

Robert liank and Giorgio Satta. 1998. Optimality Theory and the generative complexity of constraint violability. Comp. Ling., 24(2):307-315.

Bruce Hayes. 1995. Metrical Stress Theory: Principles and Case Studies. U. of Chicago Press.

C. Douglas Jolnson. 1972. Formal Aspects of Phonological Description. Mouton.

Ronald M. Kaplan and Martin Kaly. 1994. Regular models of phomological rule systems. Computational Linguistics, 20(3):331-378.

I muri Kartitunen. 1998. The proper treatment of optimality in computational phonology. In Proc. of FSMNLI'98, 1 12, Bilkont U., Ankara, Turkey.

linda Jombardi. 1999. Positional faithfulnoss and voicing assimilation in Optimality Theory. Natural Language and Linguistic Theory, 17:267--302.

John MeCarthy and Alan Prince. 1993. Generalized alignmont. In Gecrt Booij and Jaap van Marle, editors, Yearbook of Morphology, 79 153. Kluwer:

John MeCarthy and Alan Prince. 1995. Faithfulness and reduplicative identity. In Jill Beckmam et al, colitor, l'upers in Optimality Theory, 259 384. U. of Massachusetts, Amherst: GLSA.

Amnin Mester and Jayo Pudgett. 1994. Directional syllabification in Genoralized Alignmont. Phonology at Santa Cruz 3, October.

Molnyar Mohri. 1997. Finite-state transelucers in language \& speced processing. Comp. Ling. 23(2).

Alan Prince and P'aul Smolensky. 1993. Optimality Theory: Constraint interaction in generative grannmar. Ms., Rutgers U. and U. of Colorado.

Alan Prince. 1983. Relatiug to the gricl. Linguistic: Inquiry, 14:19-100.

J. P. Stemberger. 1996. 'The scope of the theory: Where does beyond lie? In L. MeNair, K. Singer, L. M. Dobrin, and M. M. Aucoin, eds. Pupers from the Parascssion on Theory and Data in Linguistics, CLS 29, 139 164. Chicago Linguistic Socicty.

Bruce Tesar. 1995. Computational Optimality Theory. Ph.D. thesis, U, of Colorado, Boulder.

Jochen Trommer. 1998. Optimal morphology. In 'T. Mark Ellison, editor, Proc. of the 4th ACL SIGPIION Workshop, Quebec, July.

Jochen Trommer. 1999. Mende tone patticns revisited: Tone mapping as local constraint evaluation. In Linguistics in Potsdam Working Papers.

Markus Walther. 1999. One-Ievel prosodic morphology. Arbeiten zur Linguistik 1. U. of Marburg. 